\title{
Article \\ Asymptotic Approximation of the Apostol-Tangent Polynomials Using Fourier Series
}

\author{
Cristina B. Corcino ${ }^{1,2}$, Baby Ann A. Damgo ${ }^{1}$, Joy Ann A. Cañete ${ }^{3}$ and Roberto B. Corcino ${ }^{1,2, *}$ \\ 1 Research Institute for Computational Mathematics and Physics, Cebu Normal University, Osmeña Boulevard, \\ Cebu City 6000, Philippines; corcinoc@cnu.edu.ph (C.B.C.); main.13001890@cnu.edu.ph (B.A.A.D.) \\ 2 Mathematics Department, Cebu Normal University, Osmeña Boulevard, Cebu City 6000, Philippines \\ 3 Department of Mathematics and Physics, Visayas State University, Baybay City 6521, Philippines; \\ joyann.canete@vsu.edu.ph \\ * Correspondence: corcinor@cnu.edu.ph
}

check for updates

Citation: Corcino, C.B.; Damgo, B.A.A.; Cañete, J.A.A.; Corcino, R.B. Asymptotic Approximation of the Apostol-Tangent Polynomials Using Fourier Series. Symmetry 2022, 14, 53. https://doi.org/10.3390/ sym 14010053

Academic Editor: Serkan Araci

Received: 22 November 2021 Accepted: 15 December 2021

Published: 1 January 2022

Publisher's Note: MDPI stays neutral with regard to jurisdictional claims in published maps and institutional affiliations.

Copyright: () 2022 by the authors Licensee MDPI, Basel, Switzerland. This article is an open access article distributed under the terms and conditions of the Creative Commons Attribution (CC BY) license (https:// creativecommons.org/licenses/by/ $4.0 /)$.

\begin{abstract}
Asymptotic approximations of the Apostol-tangent numbers and polynomials were established for non-zero complex values of the parameter $\lambda$. Fourier expansion of the Apostol-tangent polynomials was used to obtain the asymptotic approximations. The asymptotic formulas for the cases $\lambda=1$ and $\lambda=-1$ were explicitly considered to obtain asymptotic approximations of the corresponding tangent numbers and polynomials.
\end{abstract}

Keywords: tangent polynomials; Bernoulli polynomials; Euler polynomials; Genocchi polynomials; generating functions; asymptotic approximation

\section{Introduction}

The tangent polynomials $T_{n}(z)$ of a degree of $n$ with a complex argument $z$ are defined by the generating function (see $[1,2]$ ).

$$
\sum_{n=0}^{\infty} T_{n}(z) \frac{w^{n}}{n !}=\left(\frac{2}{e^{2 w}+1}\right) e^{z w}, \quad|w|<\frac{\pi}{2}
$$

These polynomials can be expressed in polynomial form as

$$
T_{n}(z)=\sum_{k=0}^{n}\left(\begin{array}{l}
n \\
k
\end{array}\right) T_{k} z^{n-k}
$$

where $T_{k}$ denotes the tangent numbers defined by

$$
\tan w=\sum_{n=0}^{\infty}(-1)^{n+1} T_{2 n+1} \frac{w^{2 n+1}}{(2 n+1) !}
$$

It is worth mentioning that tangent numbers are the odd indices of the numbers $A_{n}$ of alternating permutations known as the Euler zigzag numbers. The first few values of these numbers are as follows:

$$
T_{0}=1, \quad T_{1}=-1, \quad T_{3}=2, \quad T_{5}=-16, \quad T_{7}=272, \quad T_{9}=-7936, \quad T_{11}=353792 .
$$

Clearly, $T_{n}:=T_{n}(0)$ for $n \in \mathbb{N}$.

Several mathematicians were attracted to work on tangent polynomials because of the significant properties that they possessed in the field of mathematics and physics (see [3-6]). Analogues, explicit identities, and symmetric properties for tangent polynomials were derived in $[2,7,8]$. Some interesting Apostol analogues of the classical Bernoulli, Euler, and Genocchi polynomials were investigated by Apostol [9], Corcino, Lou, Srivastava and 
Araci (see [10-17]). These analogues are called the Apostol-Bernoulli, Apostol-Euler, and Apostol-Genocchi polynomials of order $m$ defined by the following relations, respectively, (see [18]): For $\lambda \in \mathbb{C} \backslash\{0\}$ and $m \in Z^{+}$,

$$
\begin{gathered}
\sum_{n=0}^{\infty} B_{n}^{m}(z ; \lambda) \frac{w^{n}}{n !}=\left(\frac{w}{\lambda e^{w}-1}\right)^{m} e^{w z},|w|<2 \pi \text { when } \lambda=1 \\
\text { and }|w+\log \lambda|<2 \pi \text { when } \lambda \neq 1 \\
\sum_{n=0}^{\infty} E_{n}^{m}(z ; \lambda) \frac{w^{n}}{n !}=\left(\frac{2}{\lambda e^{w}+1}\right)^{m} e^{w z},|w|<\pi \text { when } \lambda=1 \\
\text { and }|w+\log \lambda|<\pi \text { when } \lambda \neq 1 \\
\sum_{n=0}^{\infty} G_{n}^{m}(z ; \lambda) \frac{w^{n}}{n !}=\left(\frac{2 w}{\lambda e^{w}+1}\right)^{m} e^{w z},|w|<\pi \text { when } \lambda=1 \\
\text { and }|w+\log \lambda|<\pi \text { when } \lambda \neq 1
\end{gathered}
$$

when $m=1$, the above Equations (3)-(5) give the generating functions for the ApostolBernoulli, Apostol-Euler, and Apostol-Genocchi polynomials, respectively (see [19]). We extend the tangent polynomials as follows.

The Apostol-tangent polynomials $T_{n}(x ; \lambda)$ in $z$ are defined by means of the generating function

$$
\sum_{n=0}^{\infty} T_{n}(x ; \lambda) \frac{t^{n}}{n !}=\left(\frac{2}{\lambda e^{2 t}+1}\right) e^{x t},|2 t+\log \lambda|<\pi
$$

when $\lambda=1$, the Equation reduces to the tangent polynomials $T_{n}(x)=T_{n}(x ; 1)$.

Lopez and Temme [20] used the Fourier series to establish the asymptotic approximations of higher-order Bernoulli and Euler polynomials. C.B. Corcino and R.B. Corcino [21] derived the asymptotics of higher-order Genocchi polynomials by employing the method in $[19,22,23]$. In the study of Navas et al. [24], Fourier expansion is used to obtain the asymptotic estimates for Apostol-Bernoulli and Apostol-Euler polynomials. In this paper, the asymptotic expansion of Apostol-tangent polynomials is derived using the method of Navas et al. [24].

\section{Asymptotic Approximations}

Let $\mathcal{T}_{\lambda}=\left\{\frac{1}{2}[(2 k-1) \pi i-\log \lambda]: k \in \mathbb{Z}\right\}$ be the set of poles of the generating function Equation (6). The Fourier series expansion of the Apostol-tangent polynomials in terms of poles in $\mathcal{T}_{\lambda}$ is given in the following theorem:

Theorem 1. Let $\lambda \in \mathbb{C} \backslash\{0\}$. For $n \geq 1,0 \leq x \leq 1$,

$$
\begin{gathered}
\frac{T_{n}(x ; \lambda)}{n !}=\frac{2^{n+1}}{\lambda^{x / 2}} \sum_{k \in \mathbb{Z}} \frac{e^{\frac{1}{2}(2 k-1) \pi i x}}{[(2 k-1) \pi i-\log \lambda]^{n+1}} \\
\quad=\frac{1}{\lambda^{x / 2}} \sum_{k \in \mathbb{Z}} \frac{e^{\frac{1}{2}(2 k-1) \pi i x}}{\left\{\frac{1}{2}[(2 k-1) \pi i-\log \lambda]\right\}^{n+1}}
\end{gathered}
$$

where the logarithm is taken to be the principal branch.

Proof. Consider the integral $\int_{C N} f_{n}(z) d z$ where

$$
f_{n}(z)=\frac{2 e^{x z}}{\left(\lambda e^{2 z}+1\right) z^{n+1}}
$$

and the circle $C_{N}$ is a circle about the origin of radius $\left(\frac{1}{2}(2 N-1+\epsilon) \pi\right), N \in \mathbb{Z}^{+}$with $\epsilon$ being a fixed real number such that $\epsilon \pi i \pm \log \lambda \neq 0(\bmod \pi i)$. 
The function $f_{n}(z)$ has poles at $z=0$ of order $n+1$ and at $z_{k}=\frac{1}{2}[(2 k-1) \pi i-\log \lambda]$, $k \in \mathbb{Z}$. The poles $z_{k}$ are simple poles. Using the Cauchy Residue Theorem,

$$
\int_{C_{N}} f_{n}(z) d z=2 \pi i \operatorname{Res}\left(f_{n}(z), z=0\right)+2 \pi i \sum_{k \in Z, k<N} \operatorname{Res}\left(f_{n}(z), z=z_{k}\right) .
$$

We observe that, using the basic property of integration,

$$
\left|\int_{C_{N}} \frac{2 e^{x z} d z}{\left(\lambda e^{2 z}+1\right) z^{n+1}}\right| \leq \int_{C_{N}} \frac{\left|2 e^{x z}\right||d z|}{\left|\lambda e^{2 z}+1\right|\left|z^{n+1}\right|} .
$$

For $0 \leq x \leq 1,\left|\lambda e^{2 z}+1\right|>\left|\lambda e^{2 z}\right|$. Let $z=a+b i$,

$$
\frac{\left|e^{x z}\right|}{\left|\lambda e^{2 z}+1\right|}=\frac{\left|e^{x(a+b i)}\right|}{\left|\lambda e^{2 z}+1\right|}=\frac{e^{a x}}{\left|\lambda e^{2 z}+1\right|} \leq \frac{e^{x \Re(z)}}{\mid \lambda e^{2 z \mid}} \leq \frac{1}{|\lambda|} .
$$

Thus,

$$
\left|\int_{C_{N}} \frac{2 e^{x z} d z}{\left(\lambda e^{2 z}+1\right) z^{n+1}}\right| \leq \frac{2}{|\lambda|} \int_{C_{N}} \frac{|d z|}{\left|z^{n+1}\right|}=\frac{2^{n+1}}{|\lambda|(2 N-1+\epsilon) \pi))^{n}}
$$

As $N \rightarrow \infty$, the last expression goes to 0 . Hence, as $N \rightarrow \infty, n \geq 1$,

$$
\int_{C_{N}} \frac{2 e^{x z}}{\left(\lambda e^{2 z}+1\right) z^{n+1}} d z \rightarrow 0 .
$$

This implies that

$$
0=\operatorname{Res}\left(f_{n}(z), z=0\right)+\sum_{k \in Z,} \operatorname{Res}\left(f_{n}(z), z=z_{k}\right) .
$$

Now, the first residue $\operatorname{Res}\left(f_{n}(z), z=0\right)$ is given as

$$
\begin{aligned}
& \operatorname{Res}\left(f_{n}(z), z=0\right)=\lim _{z \rightarrow 0} \frac{1}{n !} \frac{d^{n}}{d z^{n}}(z-0)^{n+1} \frac{1}{z^{n+1}}\left(\frac{2 e^{x z}}{\lambda e^{2 z}+1}\right) \\
& =\lim _{z \rightarrow 0} \frac{1}{n !} \frac{d^{n}}{d z^{n}}\left(\frac{2 e^{x z}}{\lambda e^{2 z}+1}\right) \\
& =\lim _{z \rightarrow 0} \frac{1}{n !} \frac{d^{n}}{d z^{n}}\left(\sum_{l=0}^{\infty} T_{l}(x ; \lambda) \frac{z^{l}}{l !}\right) \\
& =\lim _{z \rightarrow 0} \frac{1}{n !} \sum_{l=n}^{\infty} T_{l}(x ; \lambda) \frac{z^{l-n}}{(l-n) !} .
\end{aligned}
$$

Note that the limit of each term of the expansion is 0 as $z \rightarrow 0$ except the term when $l=n$. This gives

$$
\operatorname{Res}\left(f_{n}(z), z=0\right)=\frac{T_{n}(x ; \lambda)}{n !} .
$$

On the other hand, the residue $\operatorname{Res}\left(f_{n}(z), z=z_{k}\right)$ is given by

$$
\begin{gathered}
\operatorname{Res}\left(f_{n}(z), z=z_{k}\right)=\lim _{z \rightarrow z_{k}}\left(z-z_{k}\right) \frac{1}{z^{n+1}}\left(\frac{2 e^{x z}}{\lambda e^{2 z}+1}\right) \\
=\frac{2 e^{x z_{k}}}{z_{k}^{n+1}} \lim _{z \rightarrow z_{k}}\left(\frac{z-z_{k}}{\lambda e^{2 z}+1}\right)=\frac{e^{(x-2) z_{k}}}{\lambda z_{k}^{n+1}} . \\
\text { Since } z_{k}=\frac{1}{2}[(2 k-1) \pi i-\log \lambda], \\
\operatorname{Res}\left(f_{n}(z), z=z_{k}\right)=\frac{e^{(x-2)\left\{\frac{1}{2}[(2 k-1) \pi i-\log \lambda]\right\}}}{\lambda\left\{\frac{1}{2}[(2 k-1) \pi i-\log \lambda]\right\}^{n+1}} \\
=\frac{-2^{n+1} e^{\frac{1}{2}(2 k-1) x \pi i}}{\left.\lambda^{\frac{x}{2}}[(2 k-1) \pi i-\log \lambda]\right]^{n+1}} .
\end{gathered}
$$


Combining these residues gives,

$$
0=\frac{T_{n}(x ; \lambda)}{n !}+\sum_{k \in \mathbb{Z}} \frac{-2^{n+1} e^{\frac{1}{2}(2 k-1) x \pi i}}{\lambda^{\frac{x}{2}}[(2 k-1) \pi i-\log \lambda]^{n+1}}
$$

Hence,

$$
T_{n}(x ; \lambda)=\frac{2^{n+1} n !}{\lambda^{\frac{x}{2}}} \sum_{\mathbf{k} \in \mathbb{Z}} \frac{e^{\frac{1}{2}(2 k-1) x \pi i}}{[(2 k-1) \pi i-\log \lambda]^{n+1}} .
$$

Corollary 1. Let $\lambda \in \mathbb{C} \backslash\{0\}$. For $n \geq 1$, the Fourier series of the Apostol-tangent numbers is given by

$$
\frac{T_{n}(0 ; \lambda)}{n !}=\sum_{k \in \mathbb{Z}} \frac{1}{\left[\frac{1}{2}(2 k-1) \pi i-\log \lambda\right]^{n+1}},
$$

where the logarithm is taken to be the principal branch.

Proof. This follows from Theorem 1 by taking $x=0$.

Proceeding as in [20], ordering of the poles of the generating function Equation (6) is carried out in the following lemma.

Lemma 1. Let $u_{k}=\frac{1}{2}[(2 k-1) \pi i-\log \lambda]$ with $k \in \mathbb{Z}, \lambda \in \mathbb{C} \backslash\{0\}$ and $\gamma=(\log \lambda) / 2 \pi i$, where the logarithm is taken to be the principal branch.

(a) If Im $\lambda>0$, then $0<\Re \mathfrak{e} \gamma<\frac{1}{2}$, and for $k \geq 1$,

$$
\left|u_{1}\right|<\left|u_{0}\right|<\left|u_{2}\right|<\left|u_{-1}\right|<\cdots<\left|u_{-k}\right|<\left|u_{k+2}\right|<
$$

(b) If Im $\lambda<0$, then $-\frac{1}{2}<\Re \mathfrak{e} \lambda<0$, and for $k \geq 1$,

$$
\left|u_{0}\right|<\left|u_{1}\right|<\left|u_{-1}\right|<\left|u_{2}\right|<\left|u_{-2}\right|<\cdots<\left|u_{-k}\right|<\left|u_{k+1}\right|<
$$

(c) If $\lambda>0$ (positive real number), then $\Re \mathfrak{e} \gamma=0$, and for $k \geq 1$,

$$
\begin{gathered}
\left|u_{0}\right|=\left|u_{1}\right|<\left|u_{-1}\right|=\left|u_{2}\right|<\left|u_{-2}\right|=\left|u_{3}\right|<\left|u_{-3}\right|=\left|u_{4}\right|<\cdots< \\
\left|u_{-k}\right|=\left|u_{k+1}\right|<\left|u_{-(k+1)}\right|=\left|u_{k+2}\right|<
\end{gathered}
$$

(d) If $\lambda<0$ (negative real number), then $\Re \mathfrak{e} \gamma=\frac{1}{2}$, and for $k \geq 1$,

$$
\begin{gathered}
\left|u_{1}\right|<\left|u_{0}\right|=\left|u_{2}\right|<\left|u_{-1}\right|=\left|u_{3}\right|<\left|u_{-2}\right|=\left|u_{4}\right|<\cdots< \\
\left|u_{k}\right|=\left|u_{-k+2}\right|=<
\end{gathered}
$$

Moreover, $\left|u_{k}\right| \geq \pi(|k|-1)$ if $|k| \geq 1$.

Proof. With the logarithm taken to be the principal branch, $\gamma$ (as a function of $\lambda$ ) maps $\lambda \in \mathbb{C} \backslash\{0\}$ to the strip $-\frac{1}{2}<\Re \mathfrak{e} \gamma \leq \frac{1}{2}$ (see [20]). To see this, write

$$
\gamma=\frac{\theta}{2 \pi}-i \frac{\ln |\lambda|}{2 \pi}
$$

where $\theta=\operatorname{Arg} \lambda$, from which we have

$$
\Re \mathfrak{e} \gamma=\frac{\theta}{2 \pi} \text { and } \operatorname{Im} \gamma=-\frac{\ln |\lambda|}{2 \pi} .
$$


with $-\pi<\theta \leq \pi$, we have

$$
\frac{-\pi}{2 \pi} \leq \Re \mathfrak{e} \gamma=\frac{\theta}{2 \pi} \leq \frac{\pi}{2 \pi} \Longrightarrow-\frac{1}{2}<\Re \mathfrak{e} \gamma \leq \frac{1}{2}
$$

where $\Re \mathfrak{e} \gamma=0$ when $\lambda>0$ and $\Re \mathfrak{e} \gamma=\frac{1}{2}$ when $\lambda<0$. If $\operatorname{Im} \lambda>0$, then $0<\theta<\pi$. Hence, $0<\Re \mathfrak{e} \gamma<\frac{1}{2}$. On the other hand, if $\operatorname{Im} \lambda<0$, then $-\pi<\theta<0$. Hence, $-\frac{1}{2}<\Re \mathfrak{e} \gamma<0$.

To verify the chains Equations (9)-(12), let $x=\Re \mathfrak{e} \gamma$ and $y=\operatorname{Im} \gamma$. Then for $k \in \mathbb{Z}$,

$$
\begin{aligned}
u_{k} & =\frac{1}{2}[(2 k-1) \pi i-\log (\lambda)] \\
& =\left(k-\frac{1}{2}\right) \pi i-\frac{\log (\lambda)}{2} \\
& =\pi i\left[k-\frac{1}{2}-\frac{\log (\lambda)}{2 \pi i}\right] \\
& =\pi i\left[k-\frac{1}{2}-(x+i y)\right] \\
& =\pi\left[i\left(k-\frac{1}{2}-x\right)+y\right] \\
& =\pi \sqrt{\left(k-\frac{1}{2}-x\right)^{2}+y^{2}}
\end{aligned}
$$

Now, we consider two cases:

Case 1. Im $\lambda>0$. Then $0<x<\frac{1}{2}$ and

$$
\begin{gathered}
\left|u_{0}\right|=\pi \sqrt{\left(-\frac{1}{2}-x\right)^{2}+y^{2}}=\pi \sqrt{\left(\frac{1}{2}+x\right)^{2}+y^{2}} \\
\left|u_{1}\right|=\pi \sqrt{\left(\frac{1}{2}-x\right)^{2}+y^{2}} \\
\left|u_{2}\right|=\pi \sqrt{\left(\frac{3}{2}-x\right)^{2}+y^{2}} \\
\left|u_{3}\right|=\pi \sqrt{\left(\frac{5}{2}-x\right)^{2}+y^{2}} \\
\left|u_{-1}\right|=\pi \sqrt{\left(-\frac{3}{2}-x\right)^{2}+y^{2}}=\pi \sqrt{\left(\frac{3}{2}+x\right)^{2}+y^{2}} \\
\left|u_{-2}\right|=\pi \sqrt{\left(-\frac{5}{2}-x\right)^{2}+y^{2}}=\pi \sqrt{\left(\frac{5}{2}+x\right)^{2}+y^{2}} \\
\left|u_{-3}\right|=\pi \sqrt{\left(-\frac{7}{2}-x\right)^{2}+y^{2}}=\pi \sqrt{\left(\frac{7}{2}+x\right)^{2}+y^{2}}
\end{gathered}
$$

From this, one can see that the order of magnitude of $u_{k}, k \in \mathbb{Z}$ given in Equation (9) holds.

Case 2. Im $\lambda<0$. Thus, $-\frac{1}{2}<x<0$. The chain of values of $u_{k}$ can be derived similarly, in which the order of magnitude of $u_{k}, k \in \mathbb{Z}$ given in Equation (10) holds.

Case 3. Im $\lambda=0$. This means that $\lambda$ is a real number, which is either positive or negative but not zero. Hence, we have the following subcases:

Subcase 1. If $\lambda>0$, then $\Re \mathfrak{e} \gamma=0$. For $k \geq 0$,

$$
\begin{aligned}
& \left|u_{k}\right|=\pi \sqrt{\left(K-\frac{1}{2}\right)^{2}+y^{2}} \\
& \left|u_{0}\right|=\pi \sqrt{\left(-\frac{1}{2}\right)^{2}+y^{2}}=\pi \sqrt{\left(\frac{1}{2}\right)^{2}+y^{2}}=\left|u_{1}\right| \\
& \left|u_{2}\right|=\pi \sqrt{\left(\frac{3}{2}\right)^{2}+y^{2}}=\pi \sqrt{\left(-\frac{3}{2}\right)^{2}+y^{2}}=\left|u_{-1}\right| \\
& \left|u_{3}\right|=\pi \sqrt{\left(\frac{5}{2}\right)^{2}+y^{2}}=\pi \sqrt{\left(-\frac{5}{2}\right)^{2}+y^{2}}=\left|u_{-2}\right| \\
& \left|u_{4}\right|=\pi \sqrt{\left(\frac{7}{2}\right)^{2}+y^{2}}=\pi \sqrt{\left(-\frac{7}{2}\right)^{2}+y^{2}}=\left|u_{-3}\right|
\end{aligned}
$$


and so on. Hence,

$$
\begin{gathered}
\left|u_{0}\right|=\left|u_{1}\right|<\left|u_{-1}\right|=\left|u_{2}\right|<\left|u_{-2}\right|=\left|u_{3}\right|<\left|u_{-3}\right|=\left|u_{4}\right|<\cdots< \\
\left|u_{-k}\right|=\left|u_{k+1}\right|<\left|u_{-(k+1)}\right|=\left|u_{k+2}\right|<\cdots,
\end{gathered}
$$

which is exactly (11).

Subcase 2. If $\lambda<0, \theta=\pi$, and hence, $x=\frac{1}{2}$. For $k \geq 0$,

$$
\begin{gathered}
\left|u_{k}\right|=\pi \sqrt{\left(k-\frac{1}{2}-x\right)^{2}+y^{2}} ; \text { when } x=\frac{1}{2} \\
=\pi \sqrt{(k-1)^{2}+y^{2}} \\
=\left|u_{-k+2}\right|
\end{gathered}
$$

from which it can easily be observed that

$$
\begin{gathered}
\left|u_{1}\right|<\left|u_{0}\right|=\left|u_{2}\right|<\left|u_{-1}\right|=\left|u_{3}\right|<\left|u_{-2}\right|=\left|u_{4}\right|<\cdots< \\
\left|u_{k}\right|=\left|u_{-k+2}\right|<\cdots
\end{gathered}
$$

which is exactly the chain in (12).

Moreover,

$$
\begin{aligned}
\left|u_{k}\right| & =\pi\left|k-\frac{1}{2}-\gamma\right| \\
& =\pi \sqrt{\left(k-\frac{1}{2}-x\right)^{2}+y^{2}} \\
& \geq \pi \sqrt{\left(k-\frac{1}{2}-x\right)^{2}} \\
& =\pi\left|k-\frac{1}{2}-x\right| \text { with }-\frac{1}{2} \leq x \leq \frac{1}{2} \\
& =\pi\left|k-\left(x+\frac{1}{2}\right)\right| \\
& \geq \pi\left(|k|-\left|x+\frac{1}{2}\right|\right) \\
& \geq \pi(|k|-1) .
\end{aligned}
$$

The asymptotic expansion of the Apostol-tangent numbers $T_{n}(0 ; \lambda)$ is given in the next theorem.

Theorem 2 Given $\lambda \in \mathbb{C} \backslash\{0\}$, let $H$ be a finite subset of $\mathcal{T}_{\lambda}$ satisfying

$$
\max \{|u|: u \in H\}<\min \left\{|u|: u \in \mathcal{T}_{\lambda} \backslash H\right\}:=v
$$

for all integers $n \geq 2$,

$$
\frac{T_{n}(0 ; \lambda)}{n !}=\sum_{u \in H} \frac{1}{u^{n+1}}+O\left(v^{-(n+1)}\right)
$$

Proof. Write the series (8) as $\sum_{k \in \mathbb{Z}} \frac{1}{\left(\mu_{k}\right)^{n+1}}$. By Lemma 1, we can relabel the set of poles by increasing order of magnitude as

$$
\left|\mu_{0}\right| \leq\left|\mu_{1}\right| \leq\left|\mu_{2}\right| \leq \cdots \leq\left|\mu_{M}\right| \leq \cdots
$$


Symmetry 2022, 14, 53

7 of 15

Since $\left|\mu_{k}\right| \geq \pi(|k|-1)$, for $k \geq 2$, the series is absolutely convergent for $n \geq 2$. For any $M>2$, the tail of the series is

$$
\sum_{k=M+1}^{\infty} \frac{1}{\left|\mu_{k}\right|^{n+1}}=\frac{1}{\left|\mu_{M+1}\right|^{n+1}} \sum_{k=M+1}^{\infty} \frac{\left|\mu_{M+1}\right|^{n+1}}{\left|\mu_{k}\right|^{n+1}}
$$

Since $k>M+1,\left|\frac{\mu_{M+1}}{\mu_{k}}\right|^{n+1} \leq 1$, we have $\left|\frac{\mu_{M+1}}{\mu_{k}}\right|^{n+1} \leq\left|\frac{\mu_{M+1}}{k}\right|^{2}$ for $n \geq 2$. Hence,

$$
\sum_{k=M+1}^{\infty} \frac{1}{\left|\mu_{k}\right|^{n+1}} \leq \frac{1}{\left|\mu_{M+1}\right|^{n+1}} \sum_{k=M+1}^{\infty}\left|\frac{\mu_{M+1}}{\mu_{k}}\right|^{2}
$$

Let

$$
C_{M, \lambda}=\sum_{k=M+1}^{\infty}\left|\frac{\mu_{M+1}}{\mu_{k}}\right|^{2}
$$

Then,

$$
\sum_{k=M+1}^{\infty} \frac{1}{\left|\mu_{k}\right|^{n+1}} \leq \frac{1}{\left|\mu_{M+1}\right|^{n+1}}\left[C_{M, \lambda}\right]=\frac{C_{M, \lambda}}{\left|\mu_{M+1}\right|^{n+1}}
$$

Now, consider $C_{M, \lambda}$ :

$$
\begin{gathered}
C_{M, \lambda}=\sum_{k=M+1}^{\infty}\left|\frac{\mu_{M+1}}{\mu_{k}}\right|^{2} \\
=\sum_{k=M+1}^{\infty} \frac{\left|\mu_{M+1}\right|^{2}}{\left|\mu_{k}\right|^{2}} \\
=\left|\mu_{M+1}\right|^{2} \sum_{k=M+1}^{\infty} \frac{1}{\left|\mu_{k}\right|^{2}}
\end{gathered}
$$

Since

$$
\begin{gathered}
\left|\mu_{k}\right|=\pi\left|k-\frac{1}{2}-\gamma\right| \geq \pi(|k|-1) \\
\left|\mu_{M+1}\right|=\pi\left|M+1-\frac{1}{2}-\gamma\right| \geq \pi(|M+1|-1) \\
=\pi\left|M+\frac{1}{2}-\gamma\right|
\end{gathered}
$$

Then

$$
\begin{gathered}
C_{M, \lambda}=\left|M+\frac{1}{2}-\gamma\right|^{2} \sum_{k=M+1}^{\infty} \frac{1}{\left|k-\frac{1}{2}-\gamma\right|^{2}} \\
\leq 2\left|M+\frac{1}{2}-\gamma\right|^{2} \sum_{k=M+1}^{\infty} \frac{1}{(|k|-1)^{2}} \\
\leq 2\left|M+\frac{1}{2}-\gamma\right|^{2}\left(\frac{1}{M^{2}}+\sum_{\uparrow=0}^{\infty} \frac{1}{(M+\uparrow)^{2}}\right)
\end{gathered}
$$

With

$$
\sum_{\uparrow=0}^{\infty} \frac{1}{(M+\uparrow)^{2}} \leq \int_{1}^{\infty} \frac{1}{(M+x)^{2}} d x=\frac{1}{M+1}
$$

So,

$$
\begin{aligned}
C_{M, \lambda} & \leq 2\left|M+\frac{1}{2}-\gamma\right|^{2}\left(\frac{1}{M^{2}}+\frac{1}{M+1}\right) \\
& =\frac{2\left|M+\frac{1}{2}-\gamma\right|^{2}}{M^{2}}+\frac{2\left|M+\frac{1}{2}-\gamma\right|^{2}}{M+1}
\end{aligned}
$$

Let

$$
\xi_{1}=\frac{\left|M+\frac{1}{2}-\gamma\right|^{2}}{M^{2}} \leq\left|\frac{3}{2}-\gamma\right|^{2}
$$


And

$$
\xi_{2}=\frac{\left|M+\frac{1}{2}-\gamma\right|}{M+1} \leq \frac{|M+1|+\left|-\frac{1}{2}-\gamma\right|}{|M+1|} \leq 1+\left|\frac{1}{2}+\gamma\right|
$$

Consequently,

$$
\begin{aligned}
C_{M, \lambda} & =2 \xi_{1}+\xi_{2}\left|M+\frac{1}{2}-\gamma\right| \\
\frac{C_{M, \lambda}}{\left|\mu_{M+1}\right|^{n+1}} & \leq \frac{2 \xi_{1}}{\left|\mu_{M+1}\right|^{n+1}}+\frac{\xi_{2}\left|M+\frac{1}{2}-\gamma\right|}{\left|\mu_{M+1}\right|^{n+1}}
\end{aligned}
$$

where

$$
\left|\mu_{M+1}\right|=\pi\left|M+\frac{1}{2}-\gamma\right|=\sqrt{\left(M+\frac{1}{2}-\operatorname{Re} \gamma\right)^{2}+(\operatorname{Im} \gamma)^{2}} \geq M
$$

So,

$$
\begin{aligned}
\frac{C_{M, \lambda}}{\left|\mu_{M+1}\right|^{n+1}} & \leq \frac{2\left|\frac{3}{2}-\gamma\right|^{2}}{\pi^{n+1}\left|M+\frac{1}{2}-\gamma\right|^{n+1}}+\frac{2\left(1+\left|\frac{1}{2}+\gamma\right|\right)\left|M+\frac{1}{2}-\gamma\right|}{\pi^{n+1}\left|M+\frac{1}{2}-\gamma\right|^{n+1}} \\
& \leq \frac{2\left|\frac{3}{2}-\gamma\right|^{2}}{\pi^{n+1}\left|M+\frac{1}{2}-\gamma\right|^{n+1}}+\frac{2\left(1+\left|\frac{1}{2}+\gamma\right|\right)}{\pi^{n+1}\left|M+\frac{1}{2}-\gamma\right|^{n}} \\
& \leq \frac{2\left|\frac{3}{2}-\gamma\right|^{2}}{\pi^{n+1}|M|^{n+1}}+\frac{2\left(1+\left|\frac{1}{2}+\gamma\right|\right)}{\pi^{n+1}|M|^{n}} \\
& \leq \frac{2\left|\frac{3}{2}-\gamma\right|^{2}}{\pi^{n+1}|M|^{n+1}}+\frac{2\left(1+\left|\frac{1}{2}+\gamma\right|\right)}{\pi^{n+1}|M|^{n+1}} \\
& \leq \frac{2\left|\frac{3}{2}-\gamma\right|^{2}}{\pi^{n+1}}+\frac{2\left(1+\left|\frac{1}{2}+\gamma\right|\right)}{\pi^{n+1}}
\end{aligned}
$$

We can see that $C_{M, \lambda} \rightarrow 0$ as $n \rightarrow \infty$ for $|M|>2$. Thus, the tail of the series is

$$
\sum_{k=M+1}^{\infty} \frac{1}{\left|\mu_{k}\right|^{n+1}} \rightarrow 0 \text { as } n \rightarrow \infty
$$

Moreover, for fixed $M>2$ and $n \gg 0, C_{M, \lambda}$ is bounded and independent of M. Hence, we can replace $C_{M, \lambda}$ with $C_{\lambda}$. This completes the proof of the theorem.

When $\lambda=1, \log \lambda=0$, and $u_{k}=\frac{1}{2}(2 k-1) \pi i, k \in \mathbb{Z}$. Take $H=\left\{\frac{\pi i}{2}, \frac{-\pi i}{2}\right\}$. Then $v=\frac{3 \pi}{2}$, and the ordinary tangent numbers $T_{n}=T_{n}(0 ; 1)$ satisfy

$$
\begin{aligned}
& \frac{T_{n}}{n !}=\frac{T_{n}(0 ; 1)}{n !} \\
& =\frac{1}{\left(\frac{\pi i}{2}\right)^{n+1}}+\frac{1}{\left(\frac{-\pi i}{2}\right)^{n+1}}+O\left(\left(\frac{3 \pi}{2}\right)^{-(n+1)}\right)
\end{aligned}
$$

An approximation of $T_{n}(0 ; 1)$ is given by

$$
\frac{T_{n}}{n !} \approx \frac{2^{n+1}}{(\pi i)^{n+1}}+\frac{2^{n+1}}{(-\pi i)^{n+1}}
$$

For even $n, n \geq 2$, it is known that $T_{n}=0$, which is also true when we use Equation (14). Then, we have

$$
\frac{T_{2 n}}{(2 n !)} \approx \frac{2^{2 n+1}}{(\pi i)^{2 n+1}}+\frac{2^{2 n+1}}{(-\pi i)^{2 n+1}}=0 .
$$

For odd indices,

$$
\begin{gathered}
\frac{T_{2 n-1}}{(2 n-1) !} \approx \frac{2^{2 n}}{(\pi i)^{2 n}}+\frac{2^{2 n}}{(-\pi i)^{2 n}} \\
\approx \frac{2^{2 n+1}}{(\pi i)^{2 n}} \\
T_{2 n-1} \approx \frac{(-1)^{n} 2^{2 n+1}(2 n-1) !}{\pi^{2 n}}, \quad n \geq 1 .
\end{gathered}
$$




$$
\begin{gathered}
T_{2(3)-1} \approx \frac{(-1)^{3} 2^{2(3)+1}(2(3)-1) !}{\pi^{2(3)}} \approx \frac{-2^{7} 5 !}{\pi 6} \\
T_{5} \approx-15.97688023 .
\end{gathered}
$$

This value is very close to the exact value of $T_{5}$ which is -16 .

It is proved in the next theorem that an asymptotic approximation of the Apostoltangent polynomials can be obtained from its Fourier series (Theorem 1) by choosing an appropriate subset of $\mathcal{T}_{\lambda}$.

Theorem 3. Given $\lambda \in \mathbb{C} \backslash\{0\}$, let $H$ be a finite subset of $\mathcal{T}_{\lambda}$ satisfying

$$
\max \{|u|: u \in H\}<\min \left\{|u|: u \in \mathcal{T}_{\lambda} \backslash H\right\}:=v .
$$

For all integers $n \geq 2$, we have uniformly for $x$ in a compact subset $K$ of $\mathbb{C}$,

$$
\frac{T_{n}(x ; \lambda)}{n !}=\sum_{u \in H} \frac{e^{u x}}{u^{n+1}}+O\left(\frac{e^{v|x|}}{v^{n+1}}\right),
$$

where the constant implicit in the order term depends on $\lambda, H$ and $K$. Moreover, for $n \gg 0$, this constant can be made independent of $K$, equal to the constant for the Apostol-tangent numbers, corresponding to the case $x=0$.

Proof. From the generating function in Equation (6), we have

$$
\frac{2 e^{(x+y) z}}{\lambda e^{2 z}+1}=\sum_{n=0}^{\infty} T_{n}(x+y ; \lambda) \frac{z^{n}}{n !} .
$$

The left-hand side of the equation can be written as

$$
\begin{aligned}
& \frac{2 e^{(x+y) z}}{\lambda e^{2 z}+1}=\frac{2 e^{x z} e^{y z}}{\lambda e^{2 z}+1}=\sum_{n=0}^{\infty} T_{n}(x ; \lambda) \frac{z^{n}}{n !} e^{y z} \\
& =\left(\sum_{n=0}^{\infty} T_{n}(x ; \lambda) \frac{z^{n}}{n !}\right)\left(\sum_{n=0}^{\infty} \frac{(y z)^{n}}{n !}\right) \\
& =\sum_{n=0}^{\infty} \sum_{k=0}^{n} T_{n-k}(x ; \lambda) \frac{z^{n-k}}{(n-k) !} \cdot \frac{(y z)^{k}}{k !} \cdot \frac{n !}{n !} \\
& =\sum_{n=0}^{\infty}\left(\sum_{k=0}^{n}\left(\begin{array}{c}
n \\
k
\end{array}\right) T_{n-k}(x ; \lambda) y^{k}\right) \frac{z^{n}}{n !} .
\end{aligned}
$$

Hence,

$$
T_{n}(x+y ; \lambda)=\sum_{k=0}^{n}\left(\begin{array}{c}
n \\
k
\end{array}\right) T_{n-k}(x ; \lambda) y^{k} .
$$

For $z \in \mathbb{C}$, writing $z=0+z($ here $y=z, x=0)$,

$$
\begin{gathered}
T_{n}(z ; \lambda)=\sum_{k=0}^{n}\left(\begin{array}{c}
n \\
k
\end{array}\right) T_{n-k}(0, \lambda) z^{k} \\
=\sum_{k=0}^{n} \frac{n !}{(n-k) ! k !} T_{n-k}(0, \lambda) z^{k} \\
\frac{T_{n}(z ; \lambda)}{n !}=\sum_{k=0}^{n} \frac{T_{n-k}(0, \lambda) z^{k}}{(n-k) ! k !} \\
=\sum_{k=0}^{n} \frac{T_{n-k}(0, \lambda)}{(n-k) !} \frac{z^{k}}{k !} \\
=\sum_{k=0}^{n}\left(\sum_{u \in H} \frac{1}{u^{n-k+1}}+O\left(v^{-(n-k+1)}\right)\right) \frac{z^{k}}{k !} \\
=\sum_{k=0}^{n}\left(\sum_{u \in H} \frac{1}{u^{n-k+1}} \frac{z^{k}}{k !}\right)+\sum_{k=0}^{n} O\left(v^{-(n-k+1)}\right) \frac{z^{k}}{k !}
\end{gathered}
$$


where the implicit constant $c$ in the order term is that corresponding to $z=0$ and only depends on $H$ and $\lambda$. Note also that

$$
\begin{gathered}
\left|\sum_{k=0}^{n} O\left(v^{-(n-k+1)}\right) \frac{z^{k}}{k !}\right| \leq \sum_{k=0}^{n} c v^{-(n-k+1)} \frac{\left|z^{k}\right|}{k !} \\
=c v^{-(n+1)} \sum_{k=0}^{n} \frac{v^{k}\left|z^{k}\right|}{k !} \\
\leq c v^{-(n+1)} e_{n}(v|z|),
\end{gathered}
$$

where

$$
e_{n}=\sum_{k=0}^{n} \frac{w^{k}}{k !}
$$

To complete the proof of the theorem, it remains to show that

$$
\frac{e_{n}^{*}(u z)}{u^{n+1}}=\frac{e^{u z}-e_{n}(u z)}{u^{n+1}}
$$

is bounded. Using the Mean Value Theorem (MVT) for Banach spaces (see also [20]), we have

$$
\begin{gathered}
e_{n}^{*}(w)=\frac{w^{n+1}}{(n+1) !}+\frac{w^{n+2}}{(n+2) !}+\cdots \\
=\frac{w^{n+1}}{(n+1) !}\left\{1+\frac{w}{n+2}+\frac{w^{2}}{(n+3)(n+2)}+\cdots\right\}
\end{gathered}
$$

from which

$$
\begin{aligned}
\left|e_{n}^{*}(w)\right| & \leq\left|\frac{w^{n+1}}{(n+1) !}\right|\left|1+\frac{w}{n+2}+\frac{w w^{2}}{(n+3)(n+2)}+\cdots\right| \\
& \leq \frac{|w|^{n+1}}{(n+1) !} \Re^{\Re e^{+}(w)}
\end{aligned}
$$

where $\Re \mathfrak{e}^{+}(w)=\max \{\Re \mathfrak{e}(w), 0\}$. Since $|u| \leq v$, for all $u \in H$, we have

$$
\begin{array}{r}
\frac{\left|e^{*}(u z)\right|}{\left|u^{n+1}\right|} \leq \frac{e^{|u z|}|u z|^{n+1}}{\left|u^{n+1}\right|(n+1) !} \\
=\frac{e^{|u z|}\left|z^{n+1}\right|\left|u^{n+1}\right|}{\left|u^{n+1}\right|(n+1) !} \\
\quad=\frac{e^{|u z|}|z|^{n+1}}{(n+1) !} \\
<\frac{e^{v|z|}|z|^{n+1}}{(n+1) !}
\end{array}
$$

so that

$$
\begin{gathered}
\left|\sum_{u \in H} \frac{e^{*}(u z)}{u^{n+1}}\right| \leq \sum_{u \in H} \frac{\mid e_{n}^{*}(u z)}{\left|u^{n+1}\right|} \\
\leq|H| e^{v|z|} \frac{|z|^{n+1}}{(n+1) !}
\end{gathered}
$$

where $|H|$ denotes the number of elements in $H$. We give the argument that

$$
|H| e^{v|z|} \frac{|z|^{n+1}}{(n+1) !}<c e^{v|z|} v^{-(n+1)}
$$

If

$$
|H| \frac{(v|z|)^{n+1}}{n+1}<c
$$

which certainly holds for $n \gg 0$, uniformly for $z$ in a compact subset $K \subset \mathbb{C}$. 
Corollary 2. Let $K$ be an arbitrary compact subset of $\mathbb{C}$. The tangent polynomials satisfy uniformly on $K$ the estimates

$$
\begin{aligned}
& \frac{T_{2 n}(x)}{(2 n) !}=\frac{(-1)^{n} 2^{2 n+2} \sin \left(\frac{\pi x}{2}\right)}{\pi^{2 n+1}}+O\left(\frac{e^{\frac{3 \pi}{2}|x|}}{\left(\frac{3 \pi}{2}\right)^{2 n+1}}\right) \\
& \frac{T_{2 n-1}(x)}{(2 n-1) !}=\frac{(-1)^{n} 2^{n+1} \cos \left(\frac{\pi x}{2}\right)}{\pi^{2 n}}+O\left(\frac{e^{\frac{3 \pi}{2}}|x|}{\left(\frac{3 \pi}{2}\right)^{2 n}}\right)
\end{aligned}
$$

where the implicit constant in the order term depends on the set K. Moreover, for $n \gg 0$, this constant can be made independent of $K$, equal to the constant for the tangent numbers, corresponding to the case $x=0$.

Proof. The tangent polynomials correspond to the case $\lambda=1$ so that

$$
u_{k}=\frac{1}{2}(2 k-1) \pi i, k \in \mathbb{Z} \text {. }
$$

Thus

$$
\mathcal{T}_{1}=\left\{\frac{1}{2}(2 k-1) \pi i: k \in \mathbb{Z}\right\}
$$

Taking

$$
H=\{(2 k-1) \pi i: k=-1,0\}=\left\{-\frac{\pi i}{2}, \frac{\pi i}{2}\right\},
$$

then $v=\frac{|3 \pi i|}{2}=\frac{3 \pi}{2}$. From Theorem 3,

$$
\begin{aligned}
& \frac{T_{n}(x ; 1)}{n !}=\sum_{u \epsilon H} \frac{e^{u x}}{u^{n+1}}+O\left(\frac{e^{v|x|}}{v^{n+1}}\right) \\
& =\left(\frac{e^{-\frac{\pi i x}{2}}}{\left(-\frac{\pi i}{2}\right)^{n+1}}+\frac{e^{\frac{\pi i x}{2}}}{\left(\frac{\pi i}{2}\right)^{n+1}}\right)+O\left(\frac{e^{\frac{3 \pi}{2}|x|}}{\left(\frac{3 \pi}{2}\right)^{n+1}}\right) .
\end{aligned}
$$

For even indices,

$$
\begin{aligned}
& \frac{T_{2 n}(x)}{(2 n) !}=\frac{T_{2 n}(x ; 1)}{(2 n) !}=\left(\frac{e^{-\frac{\pi i x}{2}}}{\left(-\frac{\pi i}{2}\right)^{2 n+1}}+\frac{e^{\frac{\pi i x}{2}}}{\left(\frac{\pi i}{2}\right)^{2 n+1}}\right)+O\left(\frac{e^{\frac{3 \pi}{2}|x|}}{2 n+1}\right) \\
& =\frac{2^{2 n+1} e^{\frac{\pi i x}{2}}}{(\pi i)^{2 n+1}}-\frac{2^{n+1} e^{-\frac{\pi i x}{2}}}{(\pi i)^{2 n+1}}+O\left(\frac{e^{\frac{3 \pi}{2}}|x|}{\left(\frac{3 \pi}{2}\right)^{2 n+1}}\right) \\
& =\frac{2^{2 n+1}\left(\cos \frac{\pi x}{2}+i \sin \frac{\pi x}{2}\right)-2^{n+1}\left(\cos \frac{\pi x}{2}-i \sin \frac{\pi x}{2}\right)}{(\pi i)^{2 n+1}}+O\left(\frac{e^{\frac{3 \pi}{2}}|x|}{\left(\frac{3 \pi}{2}\right)^{2 n+1}}\right) \\
& =\frac{2^{n+1}\left(\cos \frac{\pi x}{2}+i \sin \frac{\pi x}{2}-\cos \frac{\pi x}{2}+i \sin \frac{\pi x}{2}\right)}{(\pi i)^{2 n+1}}+O\left(\frac{e^{\frac{3 \pi}{2}|x|}}{\left(\frac{3 \pi}{2}\right)^{2 n+1}}\right)^{\frac{3 \pi}{2}|x|} \\
& =\frac{2^{n+1} 2 i \sin \frac{\pi x}{2}}{(\pi i)^{2 n+1}}+O\left(\frac{\left.e^{\frac{3 \pi}{2}}\right)^{2 n+1}}{2}\right) \\
& =\frac{2^{n+2} i \sin \left(\frac{\pi x}{2}\right)}{\pi^{2 n+1} i^{2 n+1}}+O\left(\frac{e^{\frac{3 \pi}{2}|x|}}{\left(\frac{3 \pi}{2}\right)^{2 n+1}}\right) \\
& =\frac{2^{2 n+2} \sin \left(\frac{\pi x}{2}\right)}{\pi^{2 n+1}(-1)^{n}}+O\left(\frac{e^{\frac{3 \pi}{2}|x|}}{\left(\frac{3 \pi}{2}\right)^{2 n+1}}\right) \\
& =\frac{(-1)^{n} 2^{2 n+2} \sin \left(\frac{\pi x}{2}\right)}{\pi^{2 n+1}}+O\left(\frac{e^{\frac{3 \pi}{2}|x|}}{\left(\frac{3 \pi}{2}\right)^{2 n+1}}\right) .
\end{aligned}
$$


For odd indices,

$$
\begin{aligned}
& \frac{T_{2 n-1}(x)}{(2 n-1) !}=\frac{T_{2 n-1}(x ; 1)}{(2 n-1) !}=\left(\frac{e^{\frac{-\pi i x}{2}}}{\left(\frac{-\pi i}{2}\right)^{2 n}}+\frac{e^{\frac{\pi i x}{2}}}{\left(\frac{\pi i}{2}\right)^{2 n}}\right)+O\left(\frac{e^{\frac{3 \pi}{2}|x|}}{\left(\frac{3 \pi}{2}\right)^{2 n}}\right) \\
& =\left(\frac{e^{\frac{-\pi i x}{2}}}{\left(\frac{\pi i}{2}\right)^{2 n}}+\frac{e^{\frac{\pi i x}{2}}}{\frac{(\pi i)^{2 n}}{2}}\right)+O\left(\frac{e^{\frac{3 \pi}{2}|x|}}{\left(\frac{3 \pi}{2}\right)^{2 n}}\right) \\
& =\left(\frac{2^{n} e^{\frac{-\pi i x}{2}}}{(\pi i)^{2 n}}+\frac{2^{n} e^{\frac{\pi i x}{2}}}{(\pi i)^{2 n}}\right)+O\left(\frac{e^{\frac{3 \pi}{2}|x|}}{2 n}\right) \\
& =\frac{2^{n}\left(\cos \frac{\pi x}{2}-i \sin \frac{\pi x}{2}\right)+2^{n}\left(\cos \frac{\pi x}{2}+i \sin \frac{\pi x}{2}\right)}{(\pi i)^{2 n}}+O\left(\frac{e^{\frac{3 \pi}{2}|x|}}{\left(\frac{3 \pi}{2}\right)^{2 n}}\right) \\
& =\frac{2^{n}\left(\cos \frac{\pi x}{2}-i \sin \frac{\pi x}{2}+\cos \frac{\pi x}{2}+i \sin \frac{\pi x}{2}\right)}{(\pi i)^{2 n}}+O\left(\frac{e^{\frac{3 \pi}{2}}|x|}{\left(\frac{3 \pi}{2}\right)^{2 n}}\right) \\
& =\frac{2^{n} 2 \cos \left(\frac{\pi x}{2}\right)}{(\pi i)^{2 n}}+O\left(\frac{e^{\frac{3 \pi}{2}}|x|}{\left(\frac{3 \pi}{2}\right)^{2 n}}\right) \\
& =\frac{2^{n+1} \cos \left(\frac{\pi x}{2}\right)}{\pi^{2 n}\left(i^{2}\right)^{n}}+O\left(\frac{e^{\frac{3 \pi}{2}}|x|}{\left(\frac{3 \pi}{2}\right)^{2 n}}\right) \\
& =\frac{(-1)^{n} 2^{n+1} \cos \left(\frac{\pi x}{2}\right)}{\pi^{2 n}}+O\left(\frac{e^{\frac{3 \pi}{2}}|x|}{\left(\frac{3 \pi}{2}\right)^{2 n}}\right) .
\end{aligned}
$$

\section{The Case When $\lambda$ Is a Negative Real Number}

When $\lambda$ is a negative real number, writing $\lambda=-|\lambda|$, the generating function in Equation (6) can be written as

$$
\frac{2 e^{x t}}{-|\lambda| e^{2 t}+1}=\sum_{n=0}^{\infty} T_{n}(x ;-|\lambda|) \frac{t^{n}}{n !}
$$

The poles of the generating function (3.1) is given by

$$
\mathcal{T}_{-|\lambda|}=\left\{\frac{1}{2}[(2 k+1) \pi i+\ln |\lambda|]: k \in \mathbb{Z}\right\} .
$$

The next theorem immediately follows from Theorem 3.

Theorem 4. Given that $\lambda$ is a negative real number, let $F$ be a finite subject of $\mathcal{T}_{-|\lambda|}$ satisfying

$$
\max \{|a|: a \in F\}<\min \left\{|a|: a \in \mathcal{T}_{-|\lambda|} \backslash F\right\}:=\xi
$$

For all integers $n \geq 2$, we have uniformly for $x$ in a compact subset $K$ of $\mathbb{C}$,

$$
\frac{T_{n}(x ; \lambda)}{n !}=\sum_{a \in F} \frac{e^{a x}}{a^{n+1}}+O\left(\frac{e^{\xi|x|}}{\xi^{n+1}}\right)
$$

where the constant implicit in the order term depends on $\lambda, F$, and $K$.

The Apostol-tangent numbers $T_{n}(0 ;-1)$ corresponding to the case $\lambda=-1$ have the generating function

$$
\frac{2}{-e^{2 t}+1}=\sum_{n=0}^{\infty} T_{n}(0 ;-1) \frac{t^{n}}{n !},
$$

The set of poles is $\mathcal{T}_{-1}=\{k \pi i: k \in \mathbb{Z} \backslash\{0\}\}$. An asymptotic formula for $T_{n}(0 ;-1)$ is given in the following theorem. 
Theorem 5. For $n \geq 3$, the Apostol-tangent numbers $T_{n}(0 ;-1)$ satisfying

$$
\frac{T_{n}(0 ;-1)}{n !}=\left(\frac{1}{(-\pi i)^{n+1}}+\frac{1}{(\pi i)^{n+1}}\right)+O\left((2 \pi)^{-(n+1)}\right) .
$$

In particular,

$$
\frac{T_{2 n-1}(0 ;-1)}{(2 n-1) !}=\frac{(-1)^{n} 2}{\pi^{2 n}}+O\left((2 \pi)^{-(2 n)}\right) .
$$

Proof. Taking $x=0, F=\{-\pi \mathrm{i}, \pi \mathrm{i}\}$ in Theorem 4 , then $\xi=2 \pi$.

Hence,

$$
\begin{gathered}
\frac{T_{n}(x ; \lambda)}{n !}=\sum_{a \in F} \frac{e^{a x}}{a^{n+1}}+O\left(\frac{e^{\xi|x|}}{\xi^{n+1}}\right) \\
\frac{T_{n}(0 ;-1)}{n !}=\left(\frac{1}{(-\pi i)^{n+1}}+\frac{1}{(\pi i)^{n+1}}\right)+O\left((2 \pi)^{-(n+1)}\right)
\end{gathered}
$$

for which Equation (19) follows. For $n \geq 2$, (21) gives $T_{2 n}(0 ;-1) \approx 0$. Indeed $T_{2 n}(0 ;-1)=0$

$\forall n \geq 1$. For $n \geq 1$,

$$
\begin{gathered}
\frac{T_{2 n-1}^{(0 ;-1)}}{(2 n-1) !}=\left(\frac{1}{(-\pi i)^{2 n}}+\frac{1}{(\pi i)^{2 n}}\right)+O\left((2 \pi)^{-(n+1)}\right) . \\
=\left(\frac{1}{(\pi i)^{2 n}}+\frac{1}{(\pi i)^{2 n}}\right)+O\left((2 \pi)^{-(n+1)}\right) \\
=\frac{2}{(\pi i)^{2 n}}+O\left((2 \pi)^{-(n+1)}\right) \\
=\left(\frac{2}{(\pi)^{2 n}(i)^{2 n}}\right)+O\left((2 \pi)^{-(n+1))}\right) \\
=\frac{(-1)^{n} 2}{(\pi)^{2 n}}+O\left((2 \pi)^{-(n+1))} .\right.
\end{gathered}
$$

Taking $n=4$,

$$
T_{7}(0 ;-1)=\frac{2(7 !)}{(\pi)^{8}} \approx 1.06233
$$

The actual value of $T_{7}(0 ;-1)=-\frac{2^{8} B_{8}}{8}=\frac{16}{15} \approx 1.06667$.

The Apostol-tangent polynomials $T_{n}(x ;-1)$ correspond to the case $\lambda=-1$. These polynomials have the generating function

$$
\frac{2 e^{x t}}{-e^{2 t}+1}=\sum_{n=0}^{\infty} T_{n}(x ;-1) \frac{t^{n}}{n !}
$$

We will prove the following theorem.

Theorem 6. Let $K$ be a compact subset of $\mathbb{C}$. The Apostol-tangent polynomials $T_{n}(x ;-1)$ satisfy uniformly on $K$ the estimates

$$
\begin{aligned}
& \frac{T_{2 n}(x ;-1)}{(2 n) !}=\frac{(-1)^{n} 2 \sin (\pi x)}{\pi^{2 n+1}}=O\left(\frac{e^{2 \pi|x|}}{(2 \pi)^{2 n+1}}\right) \\
& \frac{T_{2 n-1}(x ;-1)}{(2 n-1) !}=\frac{(-1)^{n} 2 \cos (\pi x)}{\pi^{2 n}}+O\left(\frac{e^{2 \pi|x|}}{(2 \pi)^{2 n}}\right) .
\end{aligned}
$$


Proof. Taking $\mathrm{F}=\{-\pi \mathrm{i}, \pi \mathrm{i}\}$, then $\xi=2 \pi$. Hence, it follows from Theorem 4 that

$$
\frac{T_{n}(x ;-1)}{n !}=\left(\frac{e^{\pi i x}}{(\pi i)^{n+1}}+\frac{e^{-\pi i x}}{(-\pi i)^{n+1}}\right)+O\left(\frac{e^{2 \pi|x|}}{(2 \pi)^{n+1}}\right) .
$$

For even indices,

$$
\begin{gathered}
\frac{T_{2 n}(x ;-1)}{(2 n) !}=\left(\frac{e^{\pi i x}}{(\pi i)^{2 n+1}}+\frac{e^{-\pi i x}}{(-\pi i)^{2 n+1}}\right)+O\left(\frac{e^{2 \pi|x|}}{(2 \pi)^{2 n+1}}\right) \\
=\left(\frac{e^{\pi i x}}{(\pi i)^{2 n+1}}-\frac{e^{-\pi i x}}{(\pi i)^{2 n+1}}\right)+O\left(\frac{e^{2 \pi|x|}}{(2 \pi)^{2 n+1}}\right) \\
=\left(\frac{\cos \pi x+i \sin \pi x-\cos \pi i x+i \sin \pi x}{(\pi i)^{2 n+1}}\right)+O\left(\frac{e^{2 \pi|x|}}{(2 \pi)^{2 n+1}}\right) \\
=\frac{2 i \sin \pi x}{(\pi i)^{2 n+1}}+O\left(\frac{e^{2 \pi|x|}}{(2 \pi)^{2 n+1}}\right)=\frac{2 \sin \pi x}{\pi^{2 n+1}(i)^{2 n}}+O\left(\frac{e^{2 \pi|x|}}{(2 \pi)^{2 n+1}}\right) \\
=\frac{(-1)^{n} 2 \sin (\pi x)}{\pi^{2 n+1}}+O\left(\frac{e^{2 \pi|x|}}{(2 \pi)^{2 n+1}}\right) .
\end{gathered}
$$

For odd indices,

$$
\begin{gathered}
\frac{T_{2 n-1}(x ;-1)}{(2 n-1) !}=\left(\frac{e^{\pi i x}}{(\pi i)^{2 n}}+\frac{e^{-\pi i x}}{(-\pi i)^{2 n}}\right)+O\left(\frac{e^{2 \pi|x|}}{(2 \pi)^{2 n}}\right) \\
=\left(\frac{e^{\pi i x}}{(\pi i)^{2 n}}+\frac{e^{-\pi i x}}{(\pi i)^{2 n}}\right)+O\left(\frac{e^{2 \pi|x|}}{(2 \pi)^{2 n}}\right) \\
=\left(\frac{\cos \pi x+i \sin \pi x+\cos \pi x-i \sin \pi x}{(\pi i)^{2 n}}\right)+O\left(\frac{e^{2 \pi|x|}}{(2 \pi)^{2 n}}\right) \\
=\frac{2 \cos \pi x}{(\pi i)^{2 n}}+O\left(\frac{e^{2 \pi|x|}}{(2 \pi)^{2 n}}\right) \\
=\frac{2 \cos \pi x}{\pi^{2 n}(i)^{2 n}}+O\left(\frac{e^{2 \pi|x|}}{(2 \pi)^{2 n}}\right) \\
=\frac{(-1)^{n} 2 \cos (\pi x)}{\pi^{2 n}}+O\left(\frac{e^{2 \pi|x|}}{(2 \pi)^{2 n}}\right) .
\end{gathered}
$$

\section{Conclusions and Recommendation}

The method of Navas et al. [24] is a clever way to obtain an asymptotic approximation from the Fourier series. In this paper, the method was applied to obtain asymptotic approximations of the Apostol-tangent numbers and polynomials for nonzero complex values of the parameter $\lambda$. The case when $\lambda$ is negative was explicitly considered because the poles are simply in terms of $\frac{1}{2} \ln |\lambda|$ plus odd multiples of $\frac{\pi}{2} i$. Moreover, the cases $\lambda=1$ and $\lambda=-1$ give beautiful approximations of the corresponding Tangent polynomials in terms of the sine and cosine functions depending on whether $n$ is even or odd.

The author recommends finding Fourier expansion and asymptotic approximations of higher-order Apostol-Tangent numbers and polynomials using the method employed in this paper (see also [25]). Furthermore, one may also try to consider multiple generalized Tangent polynomials and their p-adic interpolation function [26].

Author Contributions: Conceptualization, C.B.C. and R.B.C.; Formal analysis, R.B.C.; Funding acquisition, R.B.C.; Investigation, C.B.C., B.A.A.D., J.A.A.C. and R.B.C.; Methodology, C.B.C., B.A.A.D., J.A.A.C. and R.B.C.; Supervision, C.B.C. and R.B.C.; Validation, C.B.C. and R.B.C.; Writing-original draft, B.A.A.D. and J.A.A.C.; Writing-review \& editing, C.B.C. and R.B.C. All authors have read and agreed to the published version of the manuscript.

Funding: This research was funded by CNU Research Institute for Computational Mathematics and Physics (CNU-RICMP), grant number CNU-RICMP-5 and The APC was funded by CNU-RICMP-5. 


\section{Institutional Review Board Statement: Not applicable.}

Informed Consent Statement: Not applicable.

Data Availability Statement: The articles used to support the findings of this study are available from the corresponding author upon request.

Acknowledgments: The authors would like to thank the reviewers for reading and evaluating the manuscript thoroughly.

Conflicts of Interest: The authors declare that they have no conflict of interest.

\section{References}

1. Ryoo, C.S. A note on the Tangent numbers and polynomials. Adv. Stud. Theor. Phys. 2013, 7, 447-454. [CrossRef]

2. Ryoo, C.S. On the analogues of Tangent numbers and polynomials associated with p-adic integral on Zp. Appl. Math. Sci. 2013, 7, 3177-3183. [CrossRef]

3. Ryoo, C.S. A numerical investigation on the zeros of the Tangent polynomials. J. Appl. Math. Inform. 2014, 32, 315-322. [CrossRef]

4. Ryoo, C.S. Differential equations associated with Tangent numbers. J. Appl. Math. Inform. 2016, 34, 487-494. [CrossRef]

5. Ryoo, C.S. On the Twisted q-Tangent Numbers and Polynomials. Appl. Math. Sci. 2013, 7, 4935-4941. [CrossRef]

6. Yasmin, G.; Muhyi, A. Certain results of 2-variable q-generalized tangent-Apostol type polynomials. J. Math. Comput. Sci. 2020, 22, 238-251. [CrossRef]

7. Ryoo, C.S. Explicit Identities for the Generalized Tangent Polynomials. Nonlinear Anal. Differ. Equ. 2018, 6, 43-51. [CrossRef]

8. Ryoo, C.S. A note on the symmetric properties for the Tangent polynomials. Int. J. Math. Anal. 2013, 7, 2575-2581. [CrossRef]

9. Apostol, T.M. On the Lerch zeta function. Pac. J. Math. 1951, 1, 161-167. [CrossRef]

10. Corcino, C.B.; Damgo, B.; Corcino, R.B. Fourier expansions for Genocchi polynomials of higher order. J. Math. Comput. Sci. 2020, 22, 59-72. [CrossRef]

11. Luo, Q.M. Fourier expansions and integral representations for Genocchi polynomials. J. Integer Seq. 2009, 12, 09.1.4.

12. Luo, Q.M. Apostol-Euler polynomials of higher order and Gaussian hypergeometric functions. Taiwan. J. Math. 2006, 10, 917-925. [CrossRef]

13. Luo, Q.M. Extensions of the Genocchi Polynomials and their Fourier expansions and integral representations. Osaka J. Math. 2011, 48, 291-309.

14. Luo, Q.M.; Srivastava, H.M. Some relationships between the Apostol-Bernoulli and Apostol-Euler polynomials. Comput. Math Appl. 2006, 51, 631-642. [CrossRef]

15. Luo, Q.M.; Srivastava, H.M. Some generalizations of the Apostol-Bernoulli and Apostol-Euler polynomials. J. Math. Anal. Appl. 2005, 308, 290-302. [CrossRef]

16. Srivastava, H.M. Some formulas for the Bernoulli and Euler polynomials at rational arguments. Math. Proc. Camb. Philos. Soc. 2000, 129, 77-84. [CrossRef]

17. Araci, S.; Acikgoz, M. Construction of Fourier expansion of Apostol Frobenius-Euler polynomials and its application. Adv. Differ. Equ. 2018, 2018, 67. [CrossRef]

18. He, Y.; Araci, S.; Srivastava, H.M.; Abdel-Aty, M. Higher-order convolutions for Apostol-Bernoulli, Apostol-Euler and ApostolGenocchi polynomials. Mathematics 2019, 6, 329. [CrossRef]

19. Bayad, A. Fourier expansions for Apostol-Bernoulli, Apostol-Euler and Apostol-Genocchi polynomials. Math. Comp. 2011, 80, 2219-2221. [CrossRef]

20. Lopez, J.L.; Temme, N.M. Large Degree Asymptotics of Generalized Bernoulli and Euler Polynomials. J. Math. Anal. Appl. 2010, 363, 197-208. [CrossRef]

21. Corcino, C.B.; Corcino, R.B. Asymptotics of Genocchi polynomials and higher order Genocchi polynomials using residues. Afr. Mat. 2020, 31, 781-792. [CrossRef]

22. Lopez, J.L.; Temme, N.M. Hermite polynomials in asymptotic representations of generalized Bernoulli, Euler, Bessel, and Buchholz polynomials. J. Math. Anal. Appl. 1999, 239, 457-477. [CrossRef]

23. Lopez, J.L.; Temme, N.M. Uniform approximations of Bernoulli and Euler polynomials in terms of hyperbolic functions. Stud. Appl. Math. 1999, 103, 241-258. [CrossRef]

24. Navas, L.M.; Ruiz, F.J.; Varona, J.L. Asymptotic estimates for Apostol Bernoulli and Apostol-Euler polynomials. Math. Comp. 2012, 81, 1707-1722. [CrossRef]

25. Araci, S.; Acikgoz, M. Applications of Fourier Series and Zeta Functions to Genocchi Polynomials. Appl. Math. Inf. Sci. 2018, 12, 951-955. [CrossRef]

26. Araci, S.; Acikgoz, M.; Sen, E. A note on the p-Adic interpolation function for multiple generalized Genocchi numbers. Turk. J. Anal. Number Theory 2013, 1, 17-22. [CrossRef] 